IJMMS 29:2 (2002) 79-84

PII. S0161171202010475

http://ijmms.hindawi.com

(c) Hindawi Publishing Corp.

\title{
DYNAMICS OF NEWTON'S FUNCTIONS OF BARNA'S POLYNOMIALS
}

\author{
PIYAPONG NIAMSUP
}

Received 7 June 2000 and in revised form 18 January 2001

\begin{abstract}
We define Barna's polynomials as real polynomials with all real roots of which at least four are distinct. In this paper, we study the dynamics of Newton's functions of such polynomials. We also give the upper and lower bounds of the Hausdorff dimension of exceptional sets of these Newton's functions.
\end{abstract}

2000 Mathematics Subject Classification: 26A18, 39B12.

1. Introduction. Newton's method is a well-known iterative method used to locate the roots of functions. Barna, [1, 2, 3, 4], proved that for a real polynomial $P(x)$ with only simple real roots of which at least four are distinct, the exceptional set of initial points of its Newton's function $N(x)$ (the set of $x \in \mathbb{R}$ such that $N^{j}(x)$ does not converge to any root of $P$, where $N^{j}(x)$ denotes the $j$ th iterate of $N$ ) is homeomorphic to a Cantor subset of $\mathbb{R}$ which has the Lebesgue measure zero. Wong [7], generalized this result to real polynomials having all real roots (not necessarily simple) of which at least four are distinct (which will be called Barna's polynomials) by using a symbolic dynamics approach. In this paper, we will investigate the symbolic dynamics of Newton's functions of Barna's polynomials. Furthermore, we give the upper and lower bounds of the Hausdorff dimension of the exceptional sets.

\section{Symbolic dynamics of Newton's functions}

DEFINITION 2.1. A real polynomial with all real roots of which at least four are distinct is called a Barna's polynomial. Thus $P(x)$ is a Barna's polynomial if and only if

$$
P(x)=c \prod_{i=1}^{n}\left(x-r_{i}\right)^{m_{i}}
$$

where $c$ is a nonzero real constant, $r_{1}<r_{2}<\cdots<r_{n}, n \geq 4$, and $m_{i} \geq 1$ for all $1 \leq i \leq n$.

Definition 2.2. The Newton's function $N_{f}(x)$ of a function $f(x)$ is defined as

$$
N_{f}(x)=x-\frac{f(x)}{f^{\prime}(x)}
$$

where $f^{\prime}(x)$ is the derivative of $f(x)$. 
Let $P(x)=c \prod_{i=1}^{n}\left(x-r_{i}\right)^{m_{i}}$ be a Barna's polynomial and $N_{P}(x)$ be the Newton's function of $P$. The following are well-known properties of $N_{P}$ (see [1, 2, 3, 4, 7]).

(a) For each $i=1,2, \ldots, n-1$, there exists $c_{i} \in\left(r_{i}, r_{i+1}\right)$ such that $c_{i}$ is a zero of $P^{\prime}(x)$, the derivative of $P(x)$, and $\left\{c_{1}, c_{2}, \ldots, c_{n-1}\right\}$ is exactly the set of all zeros of $P^{\prime}$ which are not zeros of $P$.

(b) In each of the intervals $\left(-\infty, c_{1}\right)$ and $\left(c_{n-1},+\infty\right), N_{P}$ has exactly one critical point. If they are denoted by $a_{1}$ and $a_{n}$, respectively, then $a_{1} \in\left[r_{1}, c_{1}\right)$ and $a_{n} \in\left(c_{n-1}, r_{n}\right]$. Moreover, $N_{P}$ is monotone increasing on $\left(-\infty, a_{1}\right]$ and $\left[a_{n},+\infty\right)$ and monotone decreasing on $\left[a_{1}, c_{1}\right)$ and $\left(c_{n-1}, a_{n}\right]$.

(c) In each $\left(c_{i-1}, c_{i}\right), 2 \leq i \leq n-1, N_{P}$ has two critical points $s_{1}^{i}$ and $s_{2}^{i}$, where $s_{1}^{i}<s_{2}^{i}$. If $r_{i}$ is a multiple root of $N_{P}$, then $s_{1}^{i}<r_{i}<s_{2}^{i}$, if $r_{i}$ is a simple root, then either $r_{i}=s_{1}^{i}$, or $r_{i}=s_{2}^{i}$. The function $N_{P}$ is monotone increasing on $\left(s_{1}^{i}, s_{2}^{i}\right)$ and monotone decreasing on $\left(c_{i-1}, s_{1}^{i}\right)$ and $\left(s_{2}^{i}, c_{i}\right)$. Moreover, $N_{P}^{\prime}$ is monotone increasing on $\left(c_{i-1}, s_{1}^{i}\right)$ and monotone decreasing on $\left(s_{2}^{i}, c_{i}\right)$.

(d) $\lim _{x \rightarrow c_{i}^{-}} N_{P}(x)=-\infty$ and $\lim _{x \rightarrow c_{i}^{+}} N_{P}(x)=+\infty$, for all $1 \leq i \leq n-1$.

(e) $\lim _{k \rightarrow \infty} N_{P}^{k}(x)=r_{1}$ for all $x \in\left(-\infty, c_{1}\right)$ and $\lim _{k \rightarrow \infty} N_{P}^{k}(x)=r_{n}$ for all $x \in\left(c_{n},+\infty\right)$.

(f) For each $i=2,3, \ldots, n-1$, the interval $\left(c_{i-1}, c_{i}\right)$ contains exactly one period-two cycle of $N_{P}$, say, at $\left\{\alpha_{i}, \beta_{i}\right\}$ where $\alpha_{i}<s_{1}^{i}<s_{2}^{i}<\beta_{i}$. Also $N_{P}^{\prime}(a)<-1, N_{P}^{\prime}(b)<-1$, and $\lim _{k \rightarrow \infty} N_{P}^{k}(x)=r_{i}$ for all $x \in\left(\alpha_{i}, \beta_{i}\right)$.

Definition 2.3. Let $P(x)$ and $N_{P}(x)$ be as above. The exceptional set $\Lambda$ of $N_{P}$ is defined as the complement of the set of real numbers $x$ such that $N_{P}^{j}(x)=\infty$ for some $j \geq 0$ or $\lim _{k \rightarrow \infty} N_{P}^{k}(x)=r_{i}$ for some $1 \leq i \leq n$.

REMARK 2.4. Note that $\Lambda$ consists of points where $N_{P}^{k}$ is well defined for each $k \in \mathbb{N}$ and never converge to any $r_{i}$.

Since our main interest is on the set $\Lambda$, those points which are not in $\Lambda$ together with their preimages will be removed from $\mathbb{R}$. From this we have the following result on period-two cycle of Newton's function.

Proposition 2.5. The function $N_{P}$ has a period-two cycle at $\{\alpha, \beta\}$ such that $c_{1}<$ $\alpha<\alpha_{2}$ and $\beta_{n-1}<\beta<c_{n-1}$.

Proof. Since $\left(-\infty, c_{1}\right)$ and $\left(c_{n-1}, \infty\right)$ are not in $\Lambda$, we remove these sets together with their preimages. Let $y_{0}=N_{P}^{-1}\left(c_{1}\right)$ such that $y_{0} \in\left(\beta_{n-1}, c_{n-1}\right)$. Then $\left(y_{0},+\infty\right) \mp$ $\Lambda$ and we remove this interval. Next let $z_{1}=N_{P}^{-1}\left(y_{0}\right)$ such that $z_{1} \in\left(c_{1}, \alpha_{2}\right)$. Then $\left(-\infty, z_{1}\right) \subsetneq \Lambda$ and we remove this interval. Applying this procedure repeatedly we get two sequences of points $\left\{y_{i}\right\}_{i=1}^{\infty}$ and $\left\{z_{j}\right\}_{j=1}^{\infty}$ where

$$
\begin{gathered}
y_{i}=N_{P}^{-1}\left(z_{i}\right) \in\left(\beta_{n-1}, c_{n-1}\right), \\
\beta_{n-1}<\cdots<y_{i}<\cdots<y_{2}<y_{1}<y_{0}<c_{n-1}, \\
z_{j}=N_{P}^{-1}\left(y_{j-1}\right) \in\left(c_{1}, \alpha_{2}\right), \\
c_{1}<z_{1}<z_{2}<\cdots<z_{j}<\cdots<\alpha_{2} .
\end{gathered}
$$

Thus $\lim _{i \rightarrow \infty} y_{i}=\beta$ and $\lim _{j \rightarrow \infty} z_{j}=\alpha$ exist. As a result,

$$
N_{P}(\beta)=N_{P}\left(\lim _{i \rightarrow \infty} y_{i}\right)=\lim _{i \rightarrow \infty} N_{P}\left(y_{i}\right)=\lim _{i \rightarrow \infty} N_{P}\left(N_{P}^{-1}\left(z_{i}\right)\right)=\lim _{i \rightarrow \infty} z_{i}=\alpha .
$$


Similarly, we have $N_{P}(\alpha)=\beta$. Since $c_{1}<\alpha \leq \alpha_{2}$ and $\beta_{n-1} \leq \beta<c_{n-1}$, we get $\alpha \neq \beta$. Finally, $\alpha \neq \alpha_{2}$ because $N_{P}(\alpha)=\beta \geq \beta_{n-1}>\beta_{2}$. This completes the proof of the proposition.

For each $i=2,3, \ldots, n-1$, we have $N_{P}\left(\left(c_{i-1}, \alpha_{i}\right]\right)=\left[\beta_{i},+\infty\right)$, and $N_{P}\left(\left[\beta_{i}, c_{i}\right)\right)=$ $\left(-\infty, \alpha_{i}\right]$. Thus there exist $t_{i} \in\left(c_{i-1}, \alpha_{i}\right]$ and $u_{i} \in\left[\beta_{i}, c_{i}\right)$ such that $N_{P}\left(t_{i}\right)=\beta$ and $N_{P}\left(u_{i}\right)=\alpha$. Then $N_{P}\left(\left(c_{i-1}, t_{i}\right]\right)=[\beta,+\infty)$ and $N_{P}\left(\left[u_{i}, c_{i}\right)\right)=(-\infty, \alpha]$. Denote the $2 n-4$ intervals

$$
\left[t_{2}, \alpha_{2}\right],\left[\beta_{2}, u_{2}\right],\left[t_{3}, \alpha_{3}\right],\left[\beta_{3}, u_{3}\right], \ldots,\left[t_{n-1}, \alpha_{n-1}\right],\left[\beta_{n-1}, u_{n-1}\right]
$$

by $I_{1}, I_{2}, \ldots, I_{2 n-4}$, respectively, and let $I=\bigcup_{i=1}^{2 n-4} I_{i}$. With a similar approach used by Wong [7], we shall define the transition matrix $V$ associated to $N_{P}$. This matrix $V$ will determine the symbolic dynamics of $N_{P}$. Let $V=\left(v_{i j}\right)$ be a $(2 n-4) \times(2 n-4)$ matrix of zeros and ones defined by

$$
v_{i j}= \begin{cases}1 & \text { if } I_{i} \cap N_{P}^{-1}\left(I_{j}\right) \neq \varnothing, \\ 0 & \text { otherwise, }\end{cases}
$$

for $i, j \in\{1,2, \ldots, 2 n-4\}$. From this definition and properties of $N_{P}$, it is easily seen that $V$ is a $(2 n-4) \times(2 n-4)$ matrix built from the following $2 \times 2$ matrices:

$$
J=\left[\begin{array}{ll}
0 & 1 \\
1 & 0
\end{array}\right], \quad M=\left[\begin{array}{ll}
1 & 1 \\
0 & 0
\end{array}\right], \quad N=\left[\begin{array}{ll}
0 & 0 \\
1 & 1
\end{array}\right] .
$$

In fact $V$ can be interpreted as an $(n-2) \times(n-2)$ matrix of matrices as follows:

(1) $V_{i i}=J$ for $1 \leq i \leq n-2$,

(2) $V_{i j}=M$ for $1 \leq i \leq n-3$, for $j>i$,

(3) $V_{i j}=N$ for $2 \leq i \leq n-2$, for $j<i$.

For example, if $n=6$, then the matrix $V$ has the form

$$
\left[\begin{array}{lccc}
J & M & M & M \\
N & J & M & M \\
N & N & J & M \\
N & N & N & J
\end{array}\right] .
$$

With the same technique in [7], $V$ is irreducible and we can show that $N_{P}$ restricted to $\Lambda$ is conjugate to the one-sided shift map $\sigma$ on the set $\sum_{2 n-4}^{V}$ where

$$
\sum_{2 n-4}^{V}=\left\{s=s_{0} s_{1} \cdots s_{n} \cdots \in \Sigma_{2 n-4} \mid v_{s_{i} s_{i+1}}=1 \forall i \geq 0\right\}
$$

is the symbolic sequences space consisting of $2 n-4$ symbols (cf. [6]).

REMARK 2.6. From [6], we have card $\left(\operatorname{Per}_{k} \sigma\right)=\operatorname{Tr}\left(V^{k}\right)$, where $\operatorname{card}\left(\operatorname{Per}_{k} \sigma\right)$ denotes the number of points of period $k$ of the shift map $\sigma$ and $\operatorname{Tr}\left(V^{k}\right)$ is the trace of $V^{k}$.

REMARK 2.7. By MATHEMATICA, we compute that $\operatorname{Tr}\left(V^{k}\right)=(n-2)^{k}+(-1)^{k}(n-2)$ where $V$ is the transition matrix associated to Newton's function of a Barna's polynomial with $n$ distinct real roots. 
We summarize this section as follows.

THEOREM 2.8. Let $P(x)=c \prod_{i=1}^{n}\left(x-r_{i}\right)^{m_{i}}$ be a Barna's function and $N_{P}$ the Newton's function of $P$. Let $\Lambda$ be the exceptional set of $N_{P}$. Then $\Lambda$ is a Cantor subset of $\mathbb{R}$ and $N_{P}$ restricted to $\Lambda$ is conjugate to the one-sided shift map on $\Sigma_{2 n-4}^{V}$.

REMARK 2.9. There is some difference between our proof of Theorem 2.8 and the proof of a similar result by Wong in [7]. In our proof we use the fact that the exceptional set $\Lambda$ lies between the period-two cycle $\{\alpha, \beta\}$ as stated in Proposition 2.5 and hence we can explicitly define the transition matrix $V$.

3. Hausdorff dimension of exceptional sets. Let $\Lambda$ be the exceptional set of Newton's function of a Barna's polynomial with $n$ distinct real roots. In this section, we give the upper and lower bounds of the Hausdorff dimension of $\Lambda$. The technique we will use here is similar to the one used in [5]. We first note that $N_{P}^{-1}$ has $n-2$ branches $N_{p, i}^{-1}$ where $N_{p, i}\left(\left(c_{i}, c_{i+1}\right)\right)=\mathbb{R}$ for all $1 \leq i \leq n-2$. We will write $N_{s_{0} s_{1} \cdots s_{k-1}}^{-k}$ for the inverse $N_{P}^{-k}$ using specific branches $N_{p, s_{0}}^{-1}, N_{p, s_{1}}^{-1}, \ldots, N_{p, s_{k-1}}^{-1}$. Let the interval $I$ be the same as in the previous section. Then $I$ has $n-2$ preimages under $N_{P}$ each in the interval $\left(c_{i-1}, c_{i}\right), 2 \leq i \leq n-1$. For each $k \geq 1$, we have

$$
N_{P}^{-k}(I)=\bigcup_{s_{0}, s_{1}, \ldots, s_{k-1}=1}^{n-2} I_{s_{0} s_{1} \cdots s_{k-1}}
$$

where $I_{s_{0} s_{1} \cdots s_{k-1}}=N_{s_{0} s_{1} \cdots s_{k-1}}^{-k}(I)$. Let $\Lambda_{k}=\left\{x \mid N_{P}^{k}(x) \in I\right\}$. Then $\Lambda_{k}=N_{P}^{-k}(I)$ and $\Lambda=\bigcap_{k \geq 0} \Lambda_{k}$. Define

$$
\begin{aligned}
m_{k} & =\min \left\{\left|N_{P}^{\prime}(x)\right| \mid x \in \Lambda_{k}\right\}, \\
m & =\min \left\{\left|N_{P}^{\prime}(x)\right| \mid x \in \Lambda\right\}, \\
M_{k} & =\max \left\{\left|N_{P}^{\prime}(x)\right| \mid x \in \Lambda_{k}\right\}, \\
M & =\max \left\{\left|N_{P}^{\prime}(x)\right| \mid x \in \Lambda\right\} .
\end{aligned}
$$

REMARK 3.1. For each $k \geq 1, M_{k} \geq M_{k+1}$, and $m_{k} \leq m_{k+1}$ since $\Lambda_{k} \supset \Lambda_{k+1}$.

We now state and prove the result on the estimation of the Hausdorff dimension of $\Lambda$.

THEOREM 3.2. $\ln (n-2) / \ln M \leq \operatorname{dim} \Lambda \leq \ln (n-2) / \ln m$.

Proof. For each $k \geq 1$, let $S_{k}$ and $L_{k}$ be the lengths of the smallest and largest intervals in $\Lambda_{k}$, respectively. For each $k \geq 0$, we get

$$
-M_{k}\left|I_{s_{0} s_{1} \cdots s_{k}}\right| \leq-M_{k+1}\left|I_{s_{0} s_{1} \cdots s_{k}}\right| \leq \int_{I_{s_{0} s_{1} \cdots s_{k}}} N^{\prime}(x) d x=-\left|I_{s_{1} \cdots s_{k}}\right| \leq-S_{k} .
$$

Hence, $\left|I_{s_{0} s_{1} \cdots s_{k}}\right| \geq S_{k} / M_{k}$. By iterating, we get $\left|I_{s_{0} s_{1} \cdots s_{k+p-1}}\right| \geq S_{k} /\left(M_{k}\right)^{p}$. Similarly, we have $\left|I_{s_{0} s_{1} \cdots s_{k+p-1}}\right| \leq L_{k} /\left(m_{k}\right)^{p}$. Since $\Lambda$ is compact, any covering $\left\{U_{i}\right\}$ of $\Lambda$ can be refined to a finite cover, where each element of this cover contains exactly one $I_{s_{0} s_{1} \cdots s_{k+p-1}}$ 
for some sufficiently large $p$. Then we get

$$
\begin{aligned}
\sum\left|U_{i}\right|^{\alpha} & \geq \sum_{s_{0}, \ldots, s_{k+p-1}=1}^{n-2}\left|I_{s_{0} s_{1} \cdots s_{k+p-1}}\right|^{\alpha} \geq \sum_{s_{0}, \ldots, s_{k+p-1}=1}^{n-2}\left(\frac{S_{k}}{\left(M_{k}\right)^{p}}\right)^{\alpha} \\
& =\left(S_{k}\right)^{\alpha} \frac{(n-2)^{k+p}}{\left(M_{k}\right)^{\alpha p}}=\left(S_{k}\right)^{\alpha}(n-2)^{k}\left(\frac{n-2}{\left(M_{k}\right)^{\alpha}}\right)^{p} .
\end{aligned}
$$

If $\alpha<\ln (n-2) / \ln M_{k}$, then this diverges as $p \rightarrow \infty$, that is, as the covering gets smaller. Thus $\operatorname{dim} \Lambda \geq \ln (n-2) / \ln M_{k}$. By letting $k \rightarrow \infty$, we have $\operatorname{dim} \Lambda \geq \ln (n-2) / \ln M$. Similarly, for a given $\epsilon>0$ and for some sufficiently large $p$, there exists a covering $\left\{U_{i}\right\}_{i=1}^{(n-2)^{k+p}}$ of $\Lambda$ such that each element of the cover contains exactly one interval $I_{s_{0} s_{1} \cdots s_{k+p-1}}$ and $\left|U_{i}\right|^{1+\epsilon} \leq\left|I_{s_{0} s_{1} \cdots s_{k+p-1}}\right|$. Thus

$$
\begin{aligned}
\sum\left|U_{i}\right|^{(1+\epsilon) \alpha} & \leq \sum_{s_{0}, \ldots, s_{k+p-1}=1}^{n-2}\left|I_{s_{0} s_{1} \cdots s_{k+p-1}}\right|^{\alpha} \\
& \leq \sum_{s_{0}, \ldots, s_{k+p-1}=1}^{n-2}\left(\frac{L_{k}}{\left(m_{k}\right)^{p}}\right)^{\alpha} \\
& =\left(L_{k}\right)^{\alpha}(n-2)^{k}\left(\frac{n-2}{\left(m_{k}\right)^{\alpha}}\right)^{p}
\end{aligned}
$$

and this goes to zero as $p \rightarrow \infty$ if $\alpha>\ln (n-2) / \ln m_{k}$. Consequently, $\operatorname{dim} \Lambda \leq(1+$ $\epsilon) \ln (n-2) / \ln m_{k}$. By letting $k \rightarrow \infty$ and $\epsilon \rightarrow 0$ we have $\operatorname{dim} \Lambda \leq \ln (n-2) / \ln m$.

EXAMPLE 3.3. Let $P(x)=(x+1)(x+2)(x-1)(x-2)$ be a Barna's polynomial and $N_{P}(x)=\left(3 x^{4}-5 x^{2}-4\right) /\left(4 x^{3}-10 x\right)$ be the Newton's function of $P$. Then $N_{P}$ has three period-two cycles approximately at

$$
\begin{aligned}
& \left\{x_{0}, x_{1}\right\}=\{-1.5435941,1.5435941\} \\
& \left\{x_{2}, x_{3}\right\}=\{-1.4790145,-0.3142616\} \\
& \left\{x_{4}, x_{5}\right\}=\{0.3142616,1.4790145\}
\end{aligned}
$$

These are the only period-two cycles by Remark 2.4. From Proposition 2.5, we obtain $\left\{x_{0}, x_{1}\right\}$ by removing the sequence of points which are the successive preimages of -2 and 2. Since $\left(N_{P}^{2}\right)^{\prime}\left(x_{0}\right)=\left(N_{P}^{2}\right)^{\prime}\left(x_{1}\right)>1,\left\{x_{0}, x_{1}\right\} \in \Lambda$. Hence, in order to find the maximum and minimum values of $\left|N^{\prime}\right|$ we must also consider the values of $\left|N_{P}^{\prime}\right|$ at the preimages of $x_{0}$ and $x_{1}$. By computation, $x_{6}=N_{P}^{-1}\left(x_{0}\right) \simeq-0.2965502$ and $x_{7}=N_{P}^{-1}\left(x_{1}\right)=0.2965502$. Since $N_{P}(x)$ is an odd function, we get, by computation,

$$
\begin{aligned}
& \left|N_{P}^{\prime}\left(x_{0}\right)\right|=\left|N_{P}^{\prime}\left(x_{1}\right)\right|=\left|N_{P}^{\prime}\left(x_{6}\right)\right|=\left|N_{P}^{\prime}\left(x_{7}\right)\right| \simeq 3.8985101, \\
& \left|N_{P}^{\prime}\left(x_{2}\right)\right|=\left|N_{P}^{\prime}\left(x_{5}\right)\right| \simeq 10.2443746, \\
& \left|N_{P}^{\prime}\left(x_{3}\right)\right|=\left|N_{P}^{\prime}\left(x_{4}\right)\right| \simeq 3.4016188 .
\end{aligned}
$$


It follows that

$$
\begin{aligned}
& m=\min \left\{\left|N_{P}^{\prime}(x)\right| \mid x \in \Lambda\right\} \simeq 3.4016188, \\
& M=\max \left\{\left|N_{P}^{\prime}(x)\right| \mid x \in \Lambda\right\} \simeq 10.2443746 .
\end{aligned}
$$

Consequently, we have

$$
0.2979063 \simeq \frac{\ln 2}{\ln M} \leq \operatorname{dim} \Lambda \leq \frac{\ln 2}{\ln m} \simeq 0.5661804 .
$$

REMARK 3.4. Let $P(x)=c \prod_{i=1}^{n}\left(x-r_{i}\right)^{m_{i}}$ be a Barna's polynomial and let $N_{P}(x)$ be its Newton's function. Let $M(x)=k N_{P}(x / k), k$ is a nonzero real constant. Then $M$ is the Newton's function of Barna's polynomial of the form $Q(x)=c_{0}(x-1)^{m_{1}} \prod_{i=1}^{n-1}(x-$ $\left.r_{i} / r_{n}\right)^{m_{i}}$ and $M$ is conjugate to $N_{P}$ via the map $h(x)=k x$. As a result, $\alpha$ is a periodic point of $N_{P}$ if and only if $k \alpha$ is a periodic point of $M$ and $N_{P}^{\prime}(\alpha)$ is equal to $M^{\prime}(k \alpha)$. Consequently, dynamics of $M$ and $N_{P}$ on their exceptional sets are the same and the Hausdorff dimensions of their exceptional sets are equal. As a result, it suffices to consider the dynamics of Newton's functions of Barna's polynomials which have 1 as the largest root.

ACKNOWLedgements. The author would like to thank Professor Julian Palmore, University of Illinois at Urbana-Champaign for drawing his attention to the subject and for many helpful comments. The author is supported by the Thailand Research Fund during the preparation of this paper.

\section{REFERENCES}

[1] B. Barna, Über die Divergenzpunkte des Newtonschen Verfahrens zur Bestimmung von Wurzeln algebraischer Gleichungen. I, Publ. Math. Debrecen 3 (1953), 109-118 (German).

[2] _ U Über die Divergenzpunkte des Newtonschen Verfahrens zur Bestimmung von Wurzeln algebraischen Gleichungen. II, Publ. Math. Debrecen 4 (1956), 384-397 (German).

[3] _ Über die divergenzpunkte des Newtonschen verfahrens zur bestimmung von wurzeln algebraischer gleichungen. III, Publ. Math. Debrecen 8 (1961), 193-207 (German).

[4] _ Über die divergenzpunkte des Newtonschen verfahrens zur bestimmung von wurzeln algebraischer gleichungen. IV, Publ. Math. Debrecen 14 (1967), 91-97 (German).

[5] J. R. Kinney and T. S. Pitcher, Julia sets for certain rational functions, Adv. in Appl. Math. 9 (1988), no. 1, 51-55.

[6] S. Wiggins, Global Bifurcations and Chaos. Analytical Methods, Applied Mathematical Sciences, vol. 73, Springer-Verlag, New York, 1988.

[7] S. Wong, Newton's method and symbolic dynamics, Proc. Amer. Math. Soc. 91 (1984), no. 2, 245-253.

Piyapong niamsup: Department of Mathematics, Faculty of Science, Chiangmai UNIVERSITY, CHIANGMAI 50200, THAILAND

E-mail address: scipnmsp@cmu.chiangmai .ac.th 


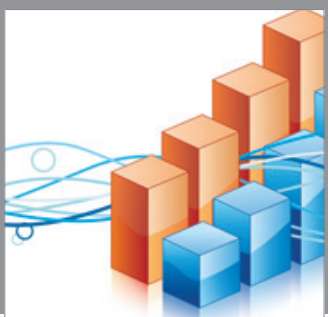

Advances in

Operations Research

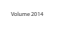

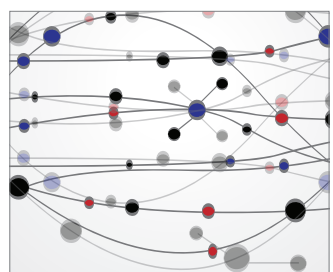

\section{The Scientific} World Journal
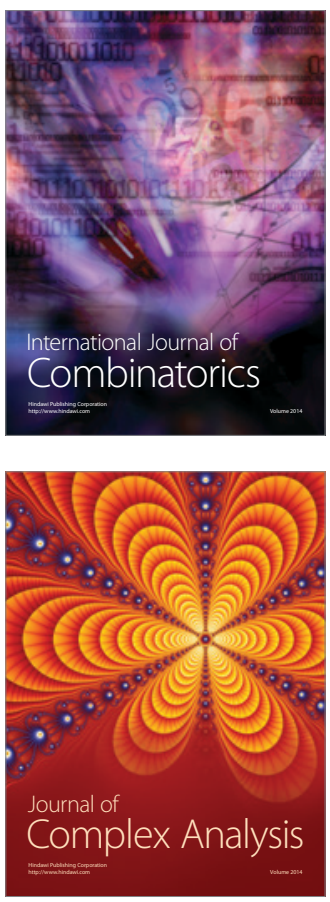

International Journal of

Mathematics and

Mathematical

Sciences
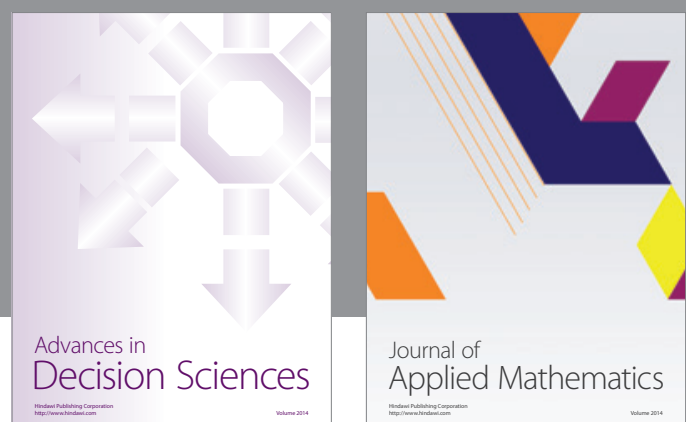

Journal of

Applied Mathematics
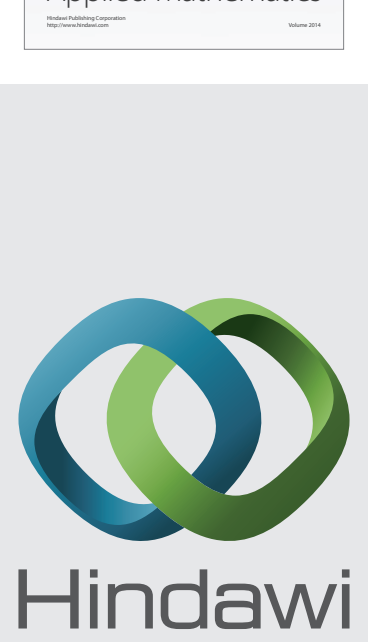

Submit your manuscripts at http://www.hindawi.com
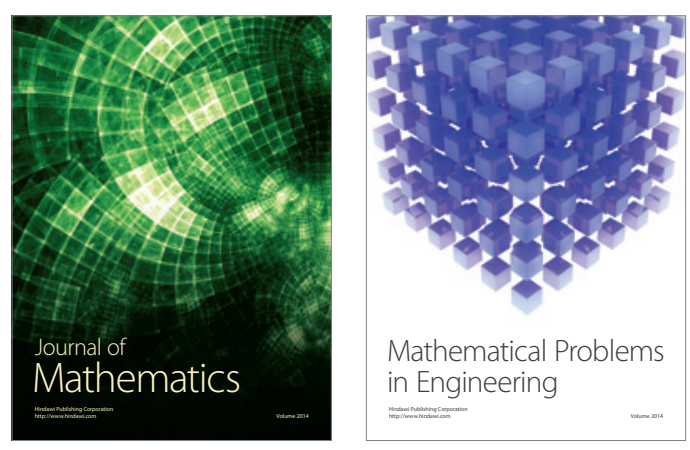

Mathematical Problems in Engineering
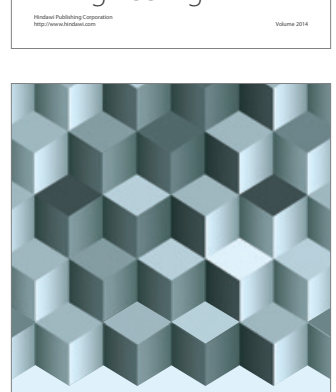

Journal of

Function Spaces
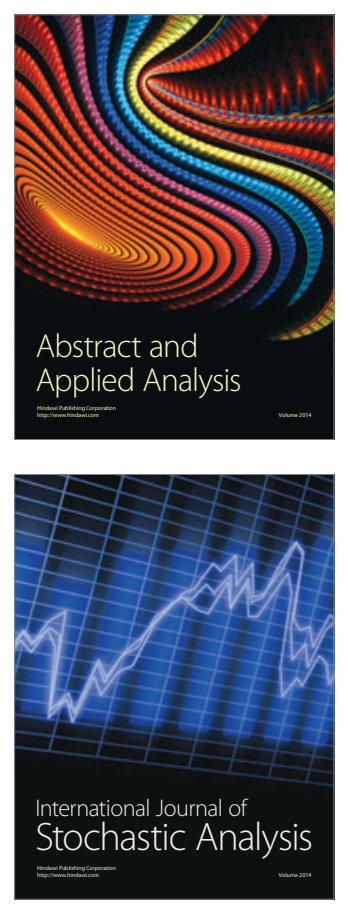

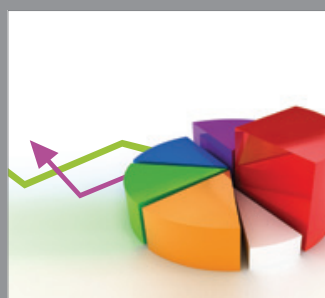

ournal of

Probability and Statistics

Promensencen
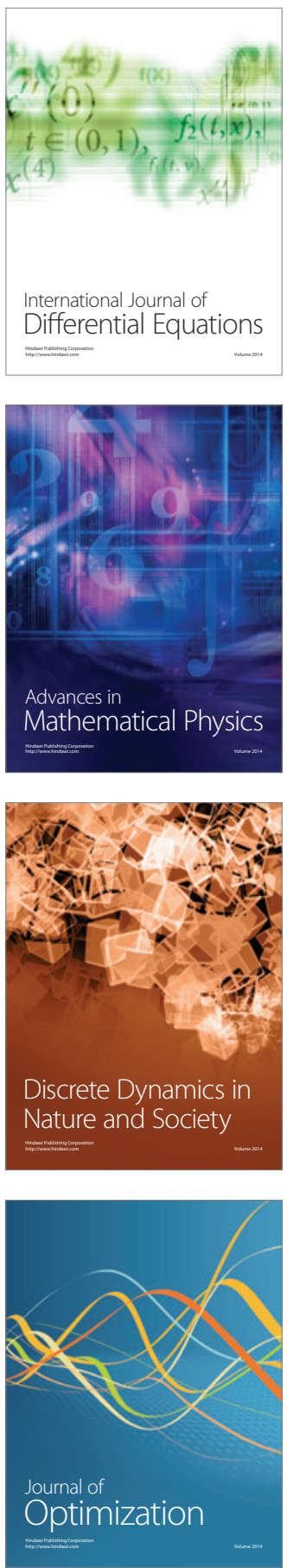